\title{
Recent Few-Body Studies at TUNL: Experimental Results and Challenges
}

\section{Thomas B. Clegg*}

University of North Carolina and Triangle Universities Nuclear Laboratory (TUNL)

Department of Physics \& Astronomy, University of North Carolina, Chapel Hill, NC-27599-3255, USA

E-mail: cleggephysics.unc.edu

A summary is provided of recent experimental studies at TUNL of proton and neutron scattering from light nuclei at incident energies below $20 \mathrm{MeV}$. The focus of these experiments is on providing new, highly accurate data for observables where significant differences exist between prior experimental results and theoretical predictions based on modern NN potentials, including chiral effective potentials.

6th International Workshop on Chiral Dynamics - CD09

Bern, Switzerland,

July 6-10 2009

${ }^{*}$ Speaker 


\section{Introduction}

My presentation today is an update on a report ${ }^{1}$ made at Chiral 2006 concerning experimental progress in low-energy few-nucleon $(\mathrm{A} \leq 4)$ scattering and reaction studies made at the Triangle Universities Nuclear Laboratory (TUNL). I am again today a spokesperson for a large number of experimental and theoretical collaborators in these recent experiments undertaken using TUNL's tandem accelerator facilities. Please note that another contributed talk at this conference by $\mathrm{H}$. Gao and the plenary talk by $\mathrm{H}$. Weller cover recent progress and planned experiments using our laboratory's highintensity gamma source $(\mathrm{HI} \gamma \mathrm{S})$.

\section{Motivation}

The essential goal of our few-nucleon program is providing new, highly-accurate measurements with incident proton or neutron energies below $20 \mathrm{MeV}$. By concentrating in this energy domain, we seek to minimize complications associated with relativistic effects and higher partial waves, thereby providing the most strenuous possible tests for increasingly detailed theoretical studies. One consequence of this low-energy approach is that strikingly good agreement is obtained between theory and experiment for most experimental observables. Our recent efforts have concentrated, therefore, on measuring observables selected to be most sensitive to theoretical-experimental differences which remain.

Modelling these few-nucleon systems theoretically has usually been based on the latest nucleon-nucleon potentials, $\left\{\right.$ eg. Nijmegen $\left.{ }^{2}, \mathrm{CD}-\mathrm{Bonn}^{3}, \mathrm{AV}-18^{4}\right\}$ supplemented by various models of three-nucleon forces ${ }^{5-8}$. These together with Fadeev methods ${ }^{9}$ are then used to calculate observables in $A=3$ and $A=4$ systems. Substantial progress has been made in assuring that increasingly complex numerical approaches are correct. An important benchmark ${ }^{10}$ showed satisfying agreement between coordinate and momentum space approaches for the $\mathrm{A}=3$ system. With that assured, recent attention has turned to $\mathrm{A}=4$ systems as a new 'theoretical laboratory.' These nuclei are the lightest with resonant states and reaction thresholds where more complete spin and isospin couplings can be studied.

Recent theoretical advances which show most promise are based on chiral potentials which treat $2 \mathrm{~N}, 3 \mathrm{~N}$ and $4 \mathrm{~N}$ interactions consistently. Figure 1 taken from a recent review by Machleidt ${ }^{11}$ depicts the hierachy of sub-nucleon interactions which are included. Twonucleon contributions appear at all orders, $3 \mathrm{~N}$ forces appear at next-to-next-to leading order $\left(\mathrm{N}^{2} \mathrm{LO}\right)$, and $4 \mathrm{~N}$ forces first

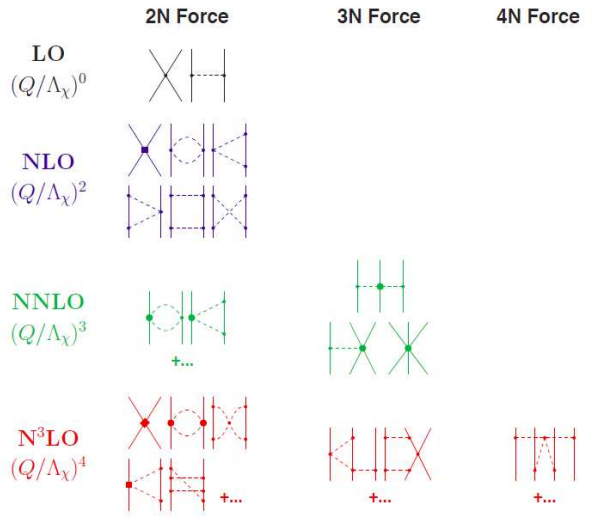

Figure 1: Hierarchy of nuclear forces in ChPT. Solid lines represent nucleons and dashed lines pions. Small dots, large solid dots, solid squares, and solid diamonds denote vertices of index $\Delta=0,1,2$, and 4 , respectively. For further explanation, see ref. 11 . 
appear at $\mathrm{N}^{3} \mathrm{LO}$. To date full theoretical formulation has appeared for $2 \mathrm{~N}$ forces ${ }^{12}$ through $\mathrm{N}^{3} \mathrm{LO}$ and for the $3 \mathrm{~N}$ interactions ${ }^{13}$ through $\mathrm{N}^{2} \mathrm{LO}$. Use of these increasingly refined potentials to calculate few-nucleon observables is well underway and results are beginning to appear.

Thus, I concentrate below on three explicit challenges where theoretical and experimental differnces are actively being explored and TUNL experimental progress has recently appeared or is anticipated.

\section{Challenge \#1: The $A_{y}$ Puzzle}

Striking differences have remained for many years between increasingly precise experimental measurements of the vector analyzing power $\mathrm{A}_{\mathrm{y}}$ in few-nucleon scattering and all theoretical predictions based on traditional $2 \mathrm{~N}$ and $3 \mathrm{~N}$ models. This has become known as the $\mathrm{A}_{\mathrm{y}}$ puzzle $^{14}$. Experimentally, $\mathrm{A}_{\mathrm{y}}$ is calculated from the left-right asymmetry of particles scattered when an incident spin-polarized beam strikes an unpolarized target. Because only the ratio of left-right counts is needed, $\mathrm{A}_{\mathrm{y}}$ can be determined much more easily and accurately than the absolute cross section, facilitating $\mathrm{A}_{\mathrm{y}}$ measurement even when its magnitude is quite small. It has been shown for light targets that $\mathrm{A}_{\mathrm{y}}$ grows with increasing target nucleon number. This occurs even at scattering energies and in systems where the long-range Coulomb interaction dominates. For example, maximum values of $A_{y}$ in p-p scattering at $5 \mathrm{MeV}^{15}$, in p-d scattering at $667 \mathrm{keV}^{16}$, and in $\mathrm{p}+{ }^{3} \mathrm{He}$ scattering at $1.6 \mathrm{MeV}^{17}$, are $0.002,0.014$, and 0.9 , respectively. More stringent tests of theory are imposed by $\mathrm{A}_{\mathrm{y}}$ measurements with incident polarized neutron beams, when the Coulomb interaction is absent. Recent TUNL measurements have concentrated on such systems.

\section{1 n-p Scattering}

Systems used recently for making the most accurate and complete $\mathrm{A}_{\mathrm{y}}$ measurements yet in n-p scattering ${ }^{18}$ are shown in Fig. 2. Neutrons of energy $12 \mathrm{MeV}$ with $70 \%$ polarization were produced via the $\mathrm{D}(\mathrm{d}, \mathrm{n})^{3} \mathrm{He}$ reaction when a polarized deuteron beam was incident on a high-pressure $\mathrm{D}_{2}$ gas target. The outgoing collimated neutron beam was directed at a NE102A plastic scintillator which served as a scattering target. A symmetric left-right array of five pairs of NE213 scintillators placed around this

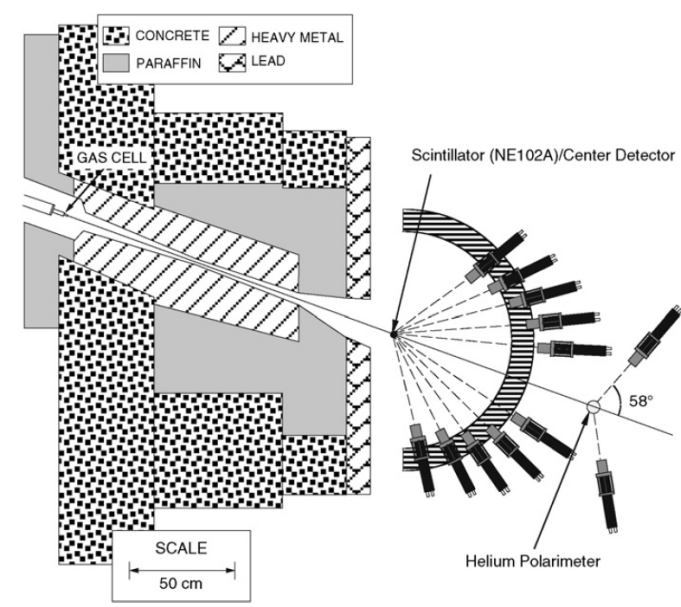

Figure 2: Experimental setup for $n-p A_{y}(\theta)$ measurements in TUNL's shielded neutron source area. target detected the angular distribution of the outgoing neutron asymmetry. Pulse-shape discrimination in these detectors reduced $\gamma$-ray backgrounds, and target-detector coincidences reduced backgrounds from neutrons scattered elsewhere. Continuous measurement of the incident neutron beam's polarization was achieved using ${ }^{4} \mathrm{He}(\mathrm{n}, \mathrm{n})$ scattering in a scintillating gas cell containing a $95 \%{ }^{4} \mathrm{He}: 5 \% \mathrm{Xe}$ mixture at 100 bar pressure. 
The $\mathrm{A}_{\mathrm{y}}$ data from this experiment were corrected for polarization dependent detector efficiencies caused by ${ }^{12} \mathrm{C}+\mathrm{n}$ scattering and then compared with theoretical calculations based on Nijmegen $93^{2}$ and CD-Bonn ${ }^{3}$ potentials. Clear need is seen for $\pi^{ \pm}$coupling which is stronger than that for $\pi^{0}$. This solidifies earlier evidence ${ }^{19,20}$ for charge symmetry breaking in the pionnucleon sector.

\section{2 n-d Scattering}

New angular distribution measurements of $A_{y}$ in the $n-d$ system have been completed at TUNL at 19 and $22.5 \mathrm{MeV}$ by a group led by G. Weisel ${ }^{21}$, but final data analysis including multiple scattering corrections is still underway. These were made to map out the evolution of $\mathrm{A}_{\mathrm{y}}$ between the $16 \mathrm{MeV}$ measurements of Sagara et al. and the $30 \mathrm{MeV}$ measurements of Dobiasch et al. Comparison of these preliminary data with theoretical predictions confirm gradual disappearance of the $\mathrm{A}_{\mathrm{y}}$ puzzle in $\mathrm{n}$-d scattering as the energy approaches $30 \mathrm{MeV}$.

\section{3. $n-{ }^{3}$ He Scattering}

A similar experimental arrangement has been employed at TUNL by J. Esterline et al. $^{22}$ to measure $\mathrm{A}_{\mathrm{y}}$ in $\mathrm{n}+{ }^{3} \mathrm{He}$ scattering at the same bombarding energies between 1.6 and $5.5 \mathrm{MeV}$ where earlier TUNL $\mathrm{p}+{ }^{3} \mathrm{He} \mathrm{A}_{\mathrm{y}}$ measurements were made by Fischer et al. ${ }^{17}$ For this experiment, the primary target was a scintillating, high-pressure gas cell similar to that used in the neutron polarimeter but with ${ }^{3} \mathrm{He}$ replacing the ${ }^{4} \mathrm{He}$. Preliminary comparisons of these new results with recent calculations of Deltuva ${ }^{23}$ based on AV-18 and CD-Bonn potentials show substantially better agreement with the new data than is observed for $\mathrm{p}+{ }^{3} \mathrm{He}$. This difference hints at what might be learned from measurements of other observables in the $A=4$ system.

\section{Challenge \#2: $\mathbf{N}+\mathrm{d}$ Breakup}

Measurements at several laboratories over the last decade have shown differences in the cross section for proton or neutron-induced breakup of a deuteron target ${ }^{24-25}$. In such a reaction, the choices of configuration for detecting the three outgoing nucleons are many. Attention has focused on the symmetric constant relative energy configuration where the three outgoing nucleons emerge in a star-configuration at $120^{\circ}$ with respect to each other and with identical relative energies in the center-of-mass system. Measurements have accumulated for two particular orientations $\alpha$ of the outgoing particle reaction plane with respect to the incident beam direction. One with $\alpha=0^{\circ}$ is the coplaner star configuration where the reaction plane includes the incident beam; the second with $\alpha=90^{\circ}$ is the space star configuration where the reaction plane is perpendicular to the incident beam.

Previous ${ }^{2} \mathrm{H}(\mathrm{n}, \mathrm{nn}) \mathrm{p}$ breakup reaction cross section measurements for these two configurations at incident neutron energies between 10 and $16 \mathrm{MeV}$ lie three standard deviations above theoretical predictions of Witala. ${ }^{25}$ Such measurements are difficult than for $\mathrm{A}_{\mathrm{y}}$ 
measurements. As in measurements those measurements mentioned above, the incident neutron beam is produced by the $\mathrm{D}(\mathrm{d}, \mathrm{n})^{3} \mathrm{He}$ reaction. However, here the incident neutron flux must be monitored using n-p elastic scattering in a $\mathrm{CH}_{2}$ target located behind the primary $\mathrm{CD}_{2}$ target. With two detectors for outgoing particles, the undetected particle scatters within a conical region determined by how outgoing energy and momentum are shared. Finite geometry corrections can be significant, and determining the absolute efficiency of the neutron detectors is challenging.

Thus, currently at TUNL we are measuring this same breakup cross section at 16 and 19 $\mathrm{MeV}$, but by detecting one neutron and the outgoing proton ${ }^{26}$. This introduces different experimental challenges and systematic uncertainties, so we expect comparison of our results with earlier data will lead both to greater confidence in the experimental results and to a better understanding of the present differences between experiment and theory.

\section{Challenge \#3: The 4N System}

Extensive cross section and $\mathrm{A}_{\mathrm{y}}$ measurements for $\mathrm{p}+{ }^{3} \mathrm{He}$ elastic scattering $(\sim 1000$ data below $12 \mathrm{MeV}$ ) and an energy-dependent phase shift analysis undertaken at Wisconsin ${ }^{27}$ could not resolve scattering phase shift uncertainties at energies below $5 \mathrm{MeV}$. Two allowed solutions emerged predicting largest differences for spin-correlation coefficients $A_{x x}$ and $A_{y y}$ below 4 $\mathrm{MeV}$. Thus, recent work at TUNL by T.V. Daniels ${ }^{28}$ sought to distinguish between these phaseshift solutions by using a polarized proton beam incident on a unique, new polarized ${ }^{3} \mathrm{He}$ $\operatorname{target}^{29}$ to make new measurements of $\mathrm{A}_{0 \mathrm{y}}, \mathrm{A}_{\mathrm{xx}}$ and $\mathrm{A}_{\mathrm{yy}}$ below $5.5 \mathrm{MeV}$.

In this experiment the ${ }^{3} \mathrm{He}$ was polarized with $\mathrm{Rb}$-spin-exchange optical pumping and then batch loaded at 1 bar to a target cell located inside a $\mu$-metal-shielded "sine-theta coil". This coil produced a transverse $0.7 \mathrm{mT}$ B-field to provide an easily reversible quantization axis for the ${ }^{3}$ He polarization. Because the scattered particle energy was low, the Pyrex target cell had thin Kapton windows over apertures for the incident beam and emerging scattered protons. A closely coupled NMR coil continuously monitored the target polarization during the experiment, and the NMR signal obtained was calibrated in a separate experiment by ${ }^{4} \mathrm{He}+{ }^{3} \mathrm{He}$ scattering. ${ }^{29}$ The target polarization lifetime was typically $\sim 2$ hours, after which the polarized ${ }^{3} \mathrm{He}$ gas was refreshed. Incident proton beam polarizations were measured using $\mathrm{p}+{ }^{4} \mathrm{He}$ scattering. Among the experimental corrections needed were asymmetries resulting from slight steering of the incident beam and scattered particles by the transverse target B-field. Largest corrections were applied at lowest energies and at forward angles.

Our new measurements of $\mathrm{A}_{0 y}, \mathrm{~A}_{\mathrm{yy}}$, and $\mathrm{A}_{\mathrm{xx}}$ at $\mathrm{E}_{\mathrm{p}}=2.28,2.77,3.15,4.02$ and $5.54 \mathrm{MeV}$ were used in combination with recent measurements of cross section and $\mathrm{A}_{\mathrm{y}}$ of Fischer et al. ${ }^{17}$ and all earlier data ( 1300 points total) in a new energy-dependent phase shift analysis. The addition of new data removes ambiguity and establishes a unique phase shift solution below 5 $\mathrm{MeV}$. These new experimental results have been compared with preliminary theoretical calculations of Viviani ${ }^{30}$ using recent chiral perturbation theory formulation of the $2 \mathrm{~N}$ 
interaction at $\mathrm{N} 3 \mathrm{LO}^{12}$ and the $3 \mathrm{~N}$ interaction at $\mathrm{N} 2 \mathrm{LO}^{13}$. Sample comparisons are shown in Fig. 3. Satisfying reduction of ' $A_{y}$ puzzle' differences are now apparent when using this recently derived version of the $3 \mathrm{~N}$ interaction. Agreement is also excellent for nuclear scattering lengths extracted from phase shifts. Our experiment provides $a_{s}=11.1 \pm 0.4 \mathrm{fm}$ and $a_{\mathrm{t}}=9.07 \pm 0.11 \mathrm{fm}$; Viviani finds $\mathrm{a}_{\mathrm{s}}=11.5 \mathrm{fm}$ and $\mathrm{a}_{\mathrm{t}}=9.13 \mathrm{fm}$. However, Machleidt suggested recently ${ }^{11}$ that sizeable three-nucleon force contributions at $\mathrm{N}^{4} \mathrm{LO}$ in the $\Delta$-less chiral perturbation theory may well be needed for full explanation of the remaining differences.
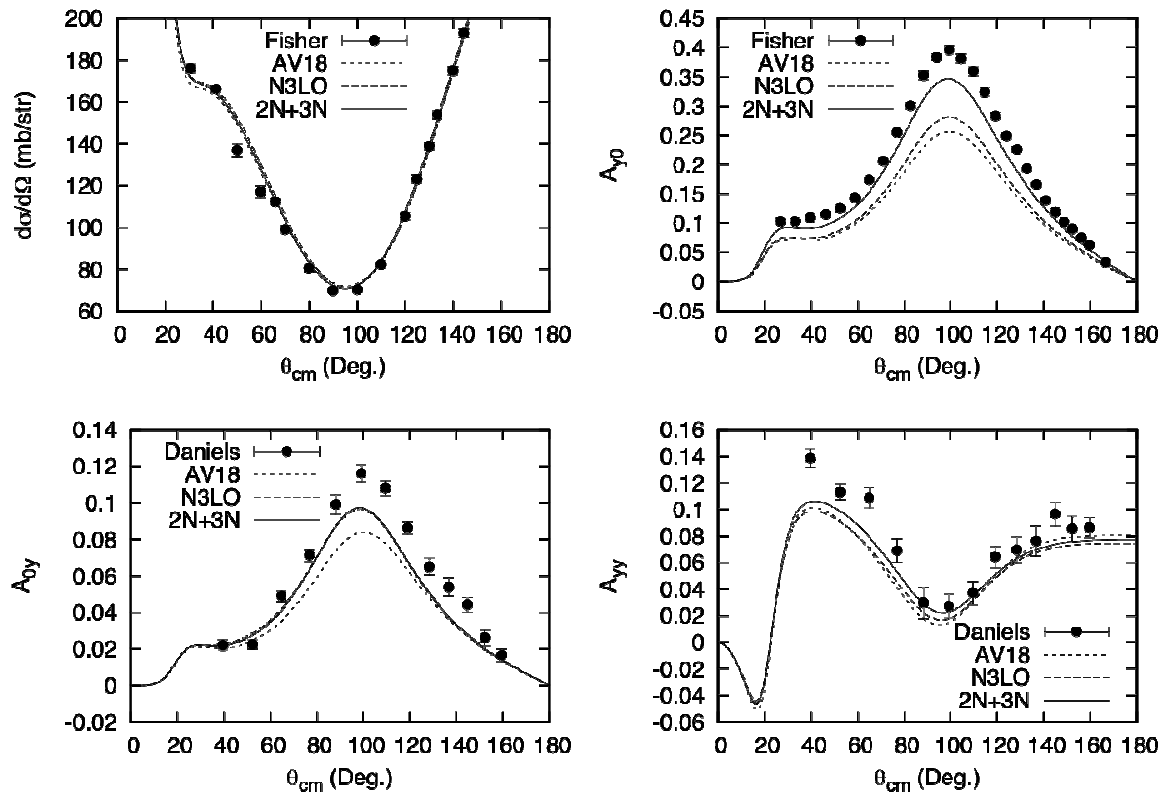

Figure 3: Comparison of TUNL cross section and $\mathrm{A}_{\mathrm{y} 0}$ data of Fisher et al. ${ }^{17}$ and the $\mathrm{A}_{0 \mathrm{y}}$ and $\mathrm{A}_{\mathrm{yy}}$ data of Daniels ${ }^{28}$ with recent theoretical calculations of Viviani ${ }^{30}$ employing separately the $2 \mathrm{~N}$ interactions of $\mathrm{AV} 18^{4}$ and $\mathrm{N}^{3} \mathrm{LO}{ }^{12}$, and the latter $2 \mathrm{~N}$ interaction combined with the $3 \mathrm{~N}$ interaction of $\mathrm{N}^{2} \mathrm{LO}^{13}$.

\section{Acknowledgement}

This work was supported in part by the US Department of Energy Office of Nuclear Physics under Grant \# DE-FG02-97ER4041.

\section{References}

[1] T.B. Clegg, $3 N$ and $4 N$ Systems and the $A_{y}$ Puzzle, in Proc. $5^{\text {th }}$ International Workshop on Chiral Dynamics, Durham/Chapel Hill, NC, USA, eds. M.W. Mohamed, H. Gao, B. Holstein, and H.R. Weller, World Scientific, 2007, pp. 350-352.

[2] V.G.J. Stoks, R.A.M Klomp, C.P.F. Terheggen, and J.J. de Swart, Construction of high quality NN potential models, Phys. Rev. C49, 2950 (1994). 
[3] R. Machleidt, The high-precision charge-dependent Bonn nucleon-nucleon potential (CD-Bonn), Phys. Rev. C 63, 024001 (2000) [nucl-th/0006014v1].

[4] R.B. Wiringa, V.G.J. Stoks, and R. Schivilla, Accurate nucleon-nucleon potential with charge independence breaking, Phys. Rev. C51, 38 (1995).

[5] B.S. Pudliner, V.R. Pandharipande, J. Carlson, S.C. Pieper, and R.B. Wiringa, Quantum Monte Carlo calculations of nuclei with A<7, Phys. Rev. C56, 1720 (1997).

[6] S.C. Pieper, V.C. Pandharipande, R.B. Wiringa, and J. Carlson, Realistic models of pion-exchange three-nucleon interactions, Phys. Rev. C64, 014001 (2001).

[7] S.A. Coon and H.K Han, Reworking the Tucson-Melbourne Three-Nucleon Potential, Few Body Syst. 30, 131 (2001).

[8] J.L. Friar, D. Hüber, and U. van Kolck, Chiral symmetry and three-nucleon forces, Phys. Rev. C59, 53 (1999).

[9] W. Glöckle, H. Witala, D. Hüber, H. Kamada, and J. Golak, The three-nucleon continuum: achievements, challenges, and applications, Physics Reports 274, 107 (1996).

[10] A. Deltuva et al., Benchmark calculation for proton-deuteron elastic scattring observables including the Coulomb interaction, Phys. Rev. C71, 064003 (2005).

[11] R. Machleidt, The Missing Three-Nucleon Forces: Where Are They? [http://arxiv.org/abs/0909.2881v1].

[12] D.R. Entem and R. Machleidt, Accurate charge-dependent nucleon-nucleon potential at fourth order of chiral perturbation theory, Phys. Rev. C68, 041001(R) (2003).

[13] V. Bernard et al., Subleading contributions to the chiral three-nucleon force: Long-range terms, Phys. Rev. C77, 064004 (2008).

[14] H. Witala, D. Hüber, and W. Glöckle, Analyzing power puzzle in low energy elastic Nd scattering, Phys. Rev. C49, R14 (1994), and references therein.

[15] M.D. Barker, P.C. Colby, and W. Haeberli, Proton-proton scattering at low energies, Phys. Rev. Lett. 48, 918 (1982).

[16] C.R. Brune, et al., Proton-deuteron scattering at low energies, Phys. Rev. C63, 044013 (2001).

[17] B.M. Fisher et al., Proton- ${ }^{3}$ He scattering at low energies, Phys. Rev. C74, 034001 (2006).

[18] R.T. Braun, et al., Neutron-proton analyzing power at $12 \mathrm{MeV}$ and inconsistencies in parametrizations of nucleon-nucleon data, Phys. Lett. B660, 161 (2008).

[19] R. Machleidt, I. Slaus, The nucleon-nucleon interaction, J. Phys. G27 (2001) R69.

[20] R. Machleidt, in Proc. Workshop on Critical Issues in the Determination of the Pion-Nucleon Coupling Constant, How sensitive are various $N N$ observables to changes in the $\pi N N$ coupling constant? Uppsala, 1999, Phys. Scr. T 87, 47 (2000).

[21] G. Weisel, et al., Neutron-Deuteron Analyzing Power $A_{y}(\theta)$ at $E_{n}=21.0 \mathrm{MeV}$, TUNL Prog. Report XLVII 2007-08, pg. 58, and references therein.

[22] J.H. Esterline, et al., Neutron- ${ }^{3} \mathrm{He}$ analyzing power between $E_{n}=1.60$ and $5.54 \mathrm{MeV}$, TUNL Progress Report XLVII 2007-08, pg. 66. 
[23] A. Deltuva, private communication.

[24] V. Huhn, et al., New investigation of the neutron-neutron and neutron-proton final-state interaction in the $n$-d breakup reaction, Phys. Rev. C63, 014003 (2000).

[25] Z. Zhou, et al., The space-star anomaly in nd breakup at $25 \mathrm{MeV}$, Nuclear Physics A684, 545c (2001).

[26] B.J. Crowe III, et al., Measurements of the Neutron-Deuteron Breakup Cross Section for the Spaceand Coplanar-Star Configurations at $19 \mathrm{MeV}$, TUNL Progress Report XLVII 2007-08, pg. 60.

[27] E. A. George and L. D. Knutson, Phys. Rev. C 67, 027001 (2003).

[28] T. V. Daniels, et al., Refinement of Global Phase-Shift Analysis for $p+{ }^{3}$ He Elastic Scattering Using Spin-Correlation Coefficients, Bull. Am. Phys. Soc. 53, no 12, (2008) p. 75.

[29] T. Katabuchi, et al., A Spin-Exchange Optically Pumped Polarized 3He Target for Low-Energy Charged Particle Scattering Experiments, Rev.Sci. Instrum. 76, 033503 (2005).

[30] M. Viviani, private communication. 